\title{
SOPORTE SOCIAL Y ESTRUCTURAS HABITACIONALES EN LA CALIDAD DE VIDA DE LAS FAMILIAS EN LA ZONA 1, COMAS 2019
}

\section{SOCIAL SUPPORT AND HOUSING STRUCTURES IN THE QUALITY OF LIFE OF FAMILIES IN ZONE 1, COMAS 2019}

\section{SUPORTE SOCIAL E ESTRUTURAS HABITACIONAIS NA QUALIDADE DE VIDA DAS FAMÍLIAS NA ZONA 1, COMAS 2019}

Lucia de Lourdes León Urraca

Universidad César Vallejo

Illu08@yahoo.com.pe

Trujillo- Perú

Yolanda Josefina Huayta Franco

Universidad César Vallejo

yolandahuaytafranco2014@gmail.com

Trujillo- Perú

Diagramación

Sindy Catherine Charcas Ibarra

Fotografia portada

Santiago Ardila Acero

Encuentre este artículo en:

http://revistas.uniminuto.edu/index.php/IYD

Para citar este artículo / To cite this article

León, L. \& Huayta, Y. (2021). Soporte social y estructuras habitacionales en la

calidad de vida de las familias en la zona 1, comas 2019. Inclusión \& Desarrollo,

$8(1)$, pp 95-105

\section{RESUMEN}

La investigación soporte social y estructuras habitacionales en la calidad de vida de las familias en la zona 1 Comas 2019 , objetivo fue determinar la incidencia del Soporte social y estructuras habitacionales en la calidad de vida de las familias en la zona 1 Comas 2019. Con enfoque cuantitativo, tipo sustantiva y diseño no experimental. Una población de 8751 familias de la zona 1 del distrito de Comas, una muestra de 368 estudiantes extraídos mediante muestro probabilístico. Se realizó la validez de los instrumentos de soporte social, estructuras habitacionales y calidad de vida, la confiabilidad tomó con los resultados y el reporte de soporte emocional fue de 0.948 , variable de estructuras habitacionales tuvo el valor de 0.986 y calidad de vida de 0.950 indicando el coeficiente de Alfa de Cronbach tiene relación muy alta y en estadística inferencial se empleó la prueba de regresión lineal múltiple. Se concluyó que, existe incidencia de las condiciones del soporte social y las 
estructuras en la calidad de vida de las familias en la zona 1 Comas, 2019, el modelo logístico es significativo $(p<0,05)$; se ajusta bien a los datos (Desviación con p<0,05); y explica el 64\% de la variable dependiente calidad de vida.

Palabras clave: soporte social, estructuras habitacionales, calidad de vida.

\section{ABSTRACT}

The research social support and housing structures in the quality of life of families in zone 1 Comas 2019, objective was to determine the incidence of social support and housing structures in the quality of life of families in zone 1 Comas 2019. With quantitative approach, substantive type and non-experimental design. A population of 8751 families in Zone 1 of the district of Comas, a sample of 368 students extracted by probabilistic sampling. The validity of social support instruments, housing structures and quality of life was realized, reliability took with the results and the report of emotional support was 0.948 , variable of housing structures had the value of 0.986 and quality of life of 0.950 indicating the Cronbach Alpha coefficient has very high relation and in inferential statistics the multiple linear regression test was used. It was concluded that, there is incidence of social support conditions and structures on the quality of life of families in zone 1 Comas, 2019 , the logistic model is significant $(p<0.05)$; it fits well with the data (Deviation with $p<0.05$ ); and explains $64 \%$ of the dependent variable quality of life.

Keywords: social support, housing structures, quality of life.

\section{SUMÀRIO}

A pesquisa suporte social e estruturas habitacionais na qualidade de vida das famílias na zona 1 Comas 2019, objetivo foi determinar a incidência do Suporte social e estruturas habitacionais na qualidade de vida das famílias na zona 1 Comas 2019. Com foco quantitativo, tipo substantivo e design não experimental. Uma população de 8751 famílias da zona 1 do distrito de Comas, uma amostra de 368 estudantes extraídos por amostragem probabilística. Realizou-se a validade dos instrumentos de suporte social, estruturas habitacionais e qualidade de vida, a confiabilidade tomou com os resultados e o relatório de suporte emocional foi de 0.948 , variável de estruturas habitacionais teve o valor de 0.986 e qualidade de vida de 0.950 indicando o coeficiente de Alfa de Cronbach tem relação muito alta e em estatística inferencial se empregou o teste de regressão linear múltipla. Concluiu-se que, existe incidência das condições do suporte social e as estruturas na qualidade de vida das famílias na zona 1 Comas, 2019, o modelo logístico é significativo $(p<0,05)$; se ajusta bem aos dados (Desvio com $p<0,05$ ); e explica $64 \%$ da variável dependente qualidade de vida.

Palavras chave: suporte social, estruturas habitacionais, qualidade de vida. 


\section{Introducción}

En el actual mundo globalizado, el crecimiento constante y acelerado de las ciudades modernas es uno de los problemas más importantes que requieren de soluciones adecuadas. Las grandes urbes de los países Latinoamericanas se encuentran planteadas bajo diversos modelos de ciudad cuyas visiones y políticas particulares de planificación fomentan construcciones informales en diferentes áreas públicas, especialmente las destinadas a las habitacionales y la falta de soporte social, cuyas consecuencias son negativas para la calidad de vida de los ciudadanos.

Al transcurso del tiempo las características de la conexión entre hombre y su naturaleza ha sufrido cambios muy marcados, que se inician desde las etapas de nuestras primeras civilizaciones, en donde se observaba que el hombre intenta habitar en estructuras que sean cada vez más confortables y que posteriormente este contexto va creciendo pero de manera inadecuada, de tal manera que hasta los tiempos posteriores marcaron un cambio por la presencia de la revolución científica, la industrialización y posterior urbanización en el cual se produjo una discordancia entre la naturaleza y el hombre imponiéndose este último de una manera incorrecta e inadmisible, deteriorándose la calidad de vida de muchas familias en el distrito de Comas.

En la política social como soporte social es una constante el tratar sobre los problemas de estructuras habitacionales de diferentes ángulos, tanto desde el enfoque social como el personal porque a mejores estructuras habitacionales mejor calidad de vida en las familias del distrito de Comas.

En países del tercer mundo las características de este tipo de asentamientos humanos, primero ocupan el suelo y luego regularizan los espacios que ocupan las familias. Este modo de ocupación del espacio es totalmente contrario en los países del primer mundo donde el proceso es más tradicional y formal. Por ese motivo, han sido catalogados como informales e ilegales que se posesionan de un determinado lugar o contratan a un informal que no realiza una buena planificación de estructuras habitacionales y por lo tanto la calidad de vida de las familias, es afectada.

La investigación se hizo en la zona 1 del distrito de Comas, en coordinación con la Municipalidad Distrital de Comas, el cual cuenta con todos los registros de los habitantes que se ven afectados en el distrito en mención, así como otros datos requeridos. El distrito de Comas cuenta con 7 zonas y es uno de los 43 distritos que conforman la provincia de Lima, ubicada en el departamento del mismo nombre en el Perú. Comas, se encuentra a unos 15 kilómetros del centro de Lima. Su altitud varía desde los 150 en sus partes más planas hasta los $811 \mathrm{msnm}$ en sus cumbres más altas Zona mayormente rural con chacras, granjas y clubes campestres. Geográficamente el distrito de Comas, cuenta con 3 tipos de lugares: (1) lugares mayormente rurales con chacras, granjas y clubes campestres, (2) lugares comerciales con mezcla de viviendas medianos recursos económicos, comprende las principales avenidas, es la planicie del distrito, (3) lugares de menores recursos económicos, calles empinadas, numerosos asentamientos humanos, ubicada en las faldas de los cerros y montañas que forman parte de la cordillera de los Andes, siendo la zona 1 parte del lugar mencionado.

En cuanto al objetivo, fue determinar la incidencia del soporte social y las condiciones de las estructuras habitacionales en la calidad de vida de las familias en la zona 1 Comas, para el año lectivo 2020. 
En cuanto a los trabajos previos internacionales, Wanzirah et al. (2017) investigó sobre la: "Estructura de la casa y riesgo de malaria en Uganda", cuyo objetivo fue reducir el riesgo de malaria al limitar la entrada de mosquitos vectores. Evaluó cómo el diseño de la casa puede afectar la entrada de mosquitos y el riesgo de malaria en Uganda. En cuanto al método se inscribieron 100 hogares en cada uno de los tres subcondados: Walukuba, distrito de Jinja; Kihihi, distrito de Kanungu; y Nagongera, distrito de Tororo. Los CDC recolectaron trampas de luz de mosquitos mensualmente en todos los hogares. Todos los niños de seis meses a diez años $(n=878)$ fueron seguidos prospectivamente durante un total de 24 meses para medir la prevalencia de parásitos cada tres meses y la incidencia de malaria. Las casas se clasificaron como modernas (paredes de cemento, madera o metal; y techo de tejas o metal; y aleros cerrados) o tradicionales (todas las demás casas). La tasa de mordedura humana fue menor en los hogares modernos que en los hogares tradicionales (tasa de incidencia ajustada (TIR) 0,48 , intervalo de confianza (IC) del 95\%: 0,37-0,64, p<0,001). Las probabilidades de infección por malaria fueron más bajas en los hogares modernos en todos los subcondados (odds ratio ajustado 0.44, IC 95\% 0.30-0.65, p <0.001), mientras que la incidencia de malaria fue más baja en los hogares modernos en Kihihi (TIR ajustada $0.61,95 \% \mathrm{Cl} 0.40-0.91, \mathrm{p}=0.02$ ) pero no en Walukuba o Nagongera. Concluyó que es probable que el diseño de la casa explique algo de la heterogeneidad de la transmisión de la malaria en Uganda y represente un objetivo prometedor para futuras intervenciones, incluso en áreas altamente endémicas.

Wu et al. (2017) presentaron un trabajo de investigación que evalúa las condiciones en cuanto a calidad de vida relacionada con la salud en adolescentes chinos, cuyo objetivo fue investigar el impacto del arreglo de vivienda en la CVRS entre los adolescentes que emigran de las escuelas rurales a las urbanas, y si el apoyo social, además de vivir con una familia, tuvo un impacto. Se realizó una encuesta transversal de 459 adolescentes escolares en dos escuelas públicas en el condado de Guyuan, Región Autónoma de Ningxia Hui, China, en 2015. De los 459 adolescentes incluidos en la muestra (de 15,41 $\pm 1,07$ años con un rango de 13 a 18 años), el $61,7 \%$ vivía con la familia y el $38,3 \%$ no vivía con la familia. Aquellos estudiantes que no viven con familias tuvieron puntajes más bajos en la Escala de Componentes Mentales (MCS) y menos apoyo social en general. Esos estudiantes, que no vivían con familias, también informaron más problemas de salud crónicos y más consumo de alcohol en comparación con los estudiantes que viven con familias. El apoyo social fue un factor mediador estadísticamente significativo sobre el efecto de los arreglos de vivienda en MCS.

En cuanto a los trabajos previos nacionales tenemos a Janampa (2018) que investigó sobre el "Diseño estructural de un edificio familiar de 5 pisos y un semisótano, Ate 2018"; el objetivo fue proporcionar una respuesta segura mientras se diseñaba y construía un edificio que pudiera dar cubrir la gran demanda residencial que hay en el Perú. Se concluye que el análisis estructural, es esencial para poder establecer el soporte del edificio, sus dimensiones, espacio, volumen y demás; este análisis debe realizarse de forma rigurosa, ya que nuestro país es altamente sísmico y el mismo va a proporcionar las bases sólidas que puedan contrarrestar los efectos sísmicos.

Maquera (2015) investigó sobre "Complejo residencial para disminuir el déficit habitacional en el distrito y provincia de Tacna", el objetivo se convirtió en asesorar una solución de oportunidad a la problemática habitacional que vive Tacna en los últimos años, en la que, debido a la rápida expansión poblacional, unida 
a la mala gestión de la ciudad y a la escasez de habitabilidad y funcionalidad urbana, genera un déficit habitacional para los hogares de estrato social medio. Es necesario incidir en la región del terreno para que articule la inspiración arquitectónica al entorno instantáneo, partiendo del análisis de los principales problemas socioeconómicos a nivel macro y micro espacial.

Las fuentes del soporte social, según Lindenthal (1971) citado por Paz (2011) propuso clases de recursos de guía social: primaria y secundaria. Primaria son aquellas que guían a las personas hacia la orientación netamente social, que comprende las relaciones de amistad dentro de la sociedad, siendo los miembros familiares y amigos cercanos, los máximos exponentes de este apoyo social a personas que se encuentran con problemas a nivel depresivos. Las fuentes secundarias, se refiere a aquellas personas que tienen una relación no tan íntima con la persona que amerita la ayuda, es decir, no es un familiar o un amigo; más bien corresponde a terapistas, psicólogos, funcionarios públicos, que pueden prestar un servicio de apoyo, de orientación y consejería.

El soporte social cuenta con un conjunto de elementos, que establecen la guía de acciones a seguir, tales como: las redes sociales, la ayuda social tal como es percibida y ayuda social que es considerada eficazmente poderosa (Stoppa Marchioro, 2012). Así mismo, Rocha (2008) manifestó que el apoyo social y la red social son estrategias con el objetivo de mejorar la calidad de vida. Ambos son ampliamente mencionados en la literatura, ya que son extensiones de los recursos individuales y se correlacionan con la prevención de efectos negativos en la salud, además de potenciar la capacidad de enfrentar situaciones adversas, lo que resulta en efectos emocionales y conductuales positivos.
Prieto (2013) manifestó que la ciudad constituye un espacio social fragmentado y segregado, condición que se evidencia en los rasgos morfológicos del hábitat. La diferenciación espacial que representan las áreas residenciales es un claro testimonio de la calidad de vida de sus habitantes.

Vivienda, para Araujo (2017) la vivienda es habitada por medio de seres humanos únicos y a través de las culturas que ellos mismos implantan, cuyas culturas son determinadas por su entorno familiar y social, desarrollándose al transcurso de su vida; lo cual los identifica en todos sus aspectos, por tanto, sus ideas con relación al tema de vivienda no se alejan de esta cultura. Rashidah, Kaur, Ahmad y Khalili (2012) encontraron que, una relación positiva pequeña, pero significativa, entre las condiciones de la vivienda, la salud, la seguridad y el apoyo social, lo que proporciona evidencia empírica de la relación entre las condiciones de la vivienda y la calidad de vida.

La residencia es un espacio cerrado, que ha sido construido para ser habitado por el ser humano; el propósito de su construcción es proporcionar refugio y espacio a los seres humanos, protegiéndolos de situaciones climáticas adversas, además, la vivienda permite que las personas tengan su privacidad y espacio particular, donde desarrollar sus actividades según sus preferencias.

La vivienda no puede ser tomada en consideración como una verdad aislada, sino como una máquina que asegura un control coherente de la ciudad con la importante interrelación entre la regulación social, la vivienda, la planificación, el urbanismo, la producción y la producción de sustancias, entre otros, bajo un conocido principio de mejora sostenible. Esta gestión integrada, este método sistémico y multidimensional, debe combinar de manera equilibrada las tres dimensiones: 
económica, social y medioambiental. Además, será probablemente un sistema multidisciplinar con una multiplicidad de actores implicados. La popularidad de una realidad heterogénea, diversa y desigual en frases de problemas, recursos, intereses y aspiraciones requiere instrucción para un buen control de las dificultades.

La vivienda básica es aquella que cumple con el concepto de germen de un círculo de núcleo familiar o de convivencia, diseñado para residir y satisfacer los deseos del ser humano, con el horizonte de que pueda alterar su composición con la incorporación de cualquier otro individuo (descendiente, pariente o huésped) o que pueda incluir áreas efectivas sin obstaculizar sus propias responsabilidades reproductivas, es decir, una vivienda que se adapte a diferentes empresas y deseos Montaner (2010).

La vivienda y su experiencia social, Haramoto (2002) expresó que la vivienda debe ser entendida como un dispositivo indispensable en el que los componentes están compuestos de tierra, infraestructura, ofertas fundamentales y equipamiento socio-comunitario dentro de un contexto dado. Aunque la vivienda sirve principalmente para satisfacer los deseos básicos de los seres humanos, también debe satisfacer las expectativas fundamentales de sus residentes, cubriendo sus necesidades primordiales que deben coincidir con su entorno ambiental y familiar.

El tema de la falta de hogar es de interés social y que transcurre en una crisis que no se ha podido solventar y que está presente desde hace algunos años. Situación que se ve agravada por una serie de problemas relacionados: pérdida de puestos de trabajo fuertes, beneficios inadecuados para acceder a una vivienda de primera categoría, resultados que se traducen en una pérdida de infraestructura fundamental, en la debilidad del sistema, en la promiscuidad de las personas, conduce mayormente a incrementar la tasa de hacinamiento, la desintegración familiar y a los problemas de salud asociados a la precarias condiciones de viviendas que no están acondicionadas para el desarrollo integral de las personas.

El saneamiento de la vivienda, lo cual se refiere a la salud que ofrece la vivienda, por tanto, las ofertas críticas o primarias de higiene y bienestar para un estilo de vida saludable y seguro en el hogar. Se describe usando el agua. Se distinguen sin duda dos criterios: la primera clase de conexión a la comunidad de agua que ingiere el hogar y el dispositivo de eliminación de aguas negras con su respectivas conexiona a las redes públicas correspondientes; y el dispositivo de saneamiento, que considera adecuado el acceso al consumo de agua fuera de la vivienda, sin embargo, dentro del terreno.

Justificación teórica, en cuanto a la variable calidad de vida según la Organización Mundial de Salud (OMS), que considera importante de los estilos de vida como creencia de una persona de su lugar en la existencia, dentro de la cultura en la que se desarrolla, del artilugio de valores que la gobierna y en estrecha relación con sus expectativas, objetivos, preocupaciones y normas. Aponte (2015).

Justificación práctica, la investigación beneficiará a la población masiva de un cuarto de Comas.

En relación con la hipótesis, existe incidencia del soporte social y las condiciones de las estructuras habitacionales en la calidad de vida de las familias en la zona 1 Comas, 2020.

Cuando nos referimos a la justificación epistemológica, según Bunge, (1960) es la filosofía de la ciencia, es la que nos propicia formas para lograr una investigación eficiente con un carácter científico, con el fin de encontrar 
nuevas verdades en el logro de un nuevo conocimiento.

Katayana, (2014) menciona a Kuhn, el cual sostiene que la visión epistemológica clásica es una especie de gradería a en torno al conocimiento de un contexto errado, contando con una investigación dinámica y ágil.

En este estudio la justificación epistemológica estará dada por las familias que habitan en la zona 1 del distrito de Comas y para ello contaremos con instrumentos como cuestionarios desarrollados con mucha amplitud sobre el tema tratado.

\section{Método}

La investigación tuvo un enfoque cuantitativo, al respecto Bernal (2016), mencionó que este tipo de estudios emplean técnicas matemáticas, pero también herramientas estadísticas porque el estudio merece este tipo de procesamiento de los resultados; en cuanto al diseño de la investigación, esta fue no experimental porque en el desarrollo y procesamiento de los resultados se operaron sin necesidad de manipular bajo ninguna circunstancia los resultados de las variables. Así también fue transversal porque toda la información que se necesitó obtener se recogió en un solo momento. También fue correlacional porque se buscó encontrar una escala de correlación o asociación con las variables y finalmente fue causal, porque trató de saber la incidencia de una variable sobre la otra.

La población estuvo conformada por 8751 familias de la zona 1 del distrito de Comas, una muestra de 368 familias comeñas, extraídas mediante muestro probabilístico aleatorio simple, a través de la fórmula para poblaciones finitas, que por cierto contó con criterios de inclusión, por consiguiente, se incluyó a un representante, varón o mujer, jefe de familia, por cada familia afectada de la zona 1 del distrito de Comas.
El método utilizado fue el hipotético deductivo de acuerdo con Sánchez y Reyes (2015) dicho método parte de hipótesis como consecuencias de los supuestos que se realizan para arribar a la hipótesis mediante los procesos inductivos.

Se empleó como técnica la encuesta, y como instrumento los cuestionarios de preguntas estructuradas, uno por cada variable, que fueron validados y fiables siendo aplicables y excelentes de acuerdo a los resultados obtenidos, en base a la puntuación del Alpha de Conbrach, previo a su aplicación.

Los valores obtenidos fueron contrastados con el sistema cuantitativo estadístico, programa de software estadístico SPSS versión 25 con el propósito de desarrollar la estadística descriptiva e inferencial para elaborar la experimentación de hipótesis con la regresión ordinal simple para localizar si los ítems de la variante soporte social, estructuras habitacionales y calidad de la vida mantienen una correlación. Según Hernández et al. (2014). Se eligió la regresión ordinal simple, a través de los resultados de la prueba de normalidad de Kolmogorov Smirnov, obteniendo una distribución de datos no normal, es decir, utilizar una prueba no paramétrica.

\section{Resultados}

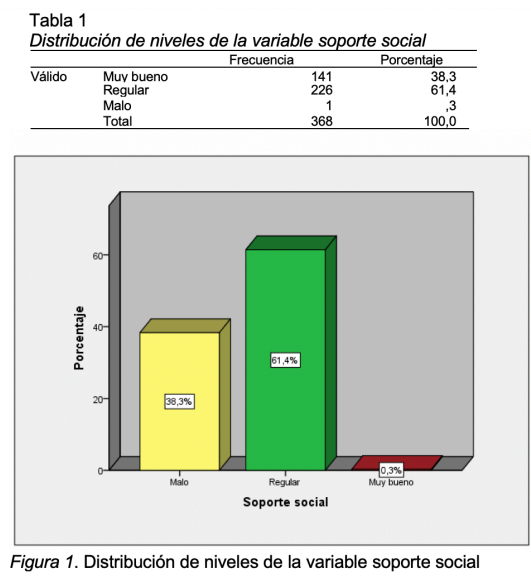


En la tabla 1 y figura 1 , se presentaron los niveles percibidos por los pobladores del distrito de Comas con respecto a la variable soporte social, el $38,3 \%$ señaló como malo, el $61,4 \%$ de regular y por último el $0,3 \%$ muy bueno. En tal sentido las familias de esta zona evidencian un nivel regular sobre el soporte social, cuyas redes sociales y amicales están arraigadas a formar parte de ellas, siendo apoyo socioemocional a los integrantes de las familias encuestadas.

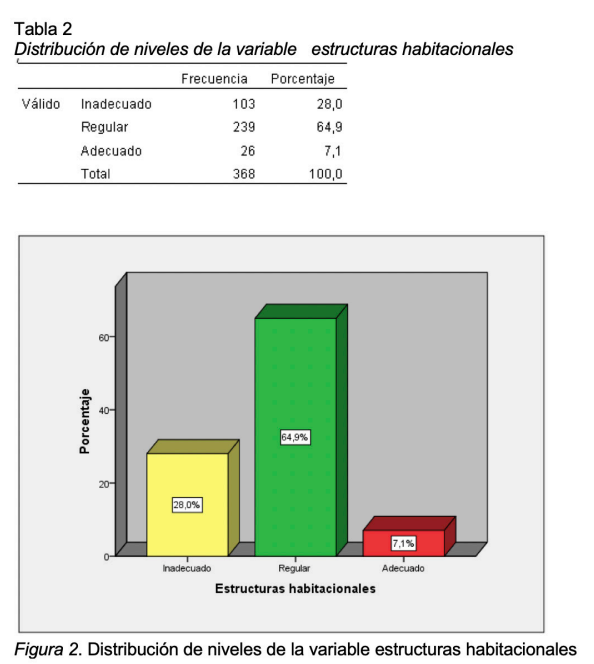

En la tabla 2 y figura 2, se presentaron las escalas percibidos por los pobladores del distrito de Comas con respecto a la variable estructura habitacionales, el $28 \%$ señaló como inadecuado, el $64.9 \%$ de regular y por último el $7.1 \%$ adecuado. Para los pobladores de la zona 1, la construcción de las estructuras habitacionales está en un nivel regular, pues cabe señalar que las construcciones aún están en proceso, con el uso de material noble.

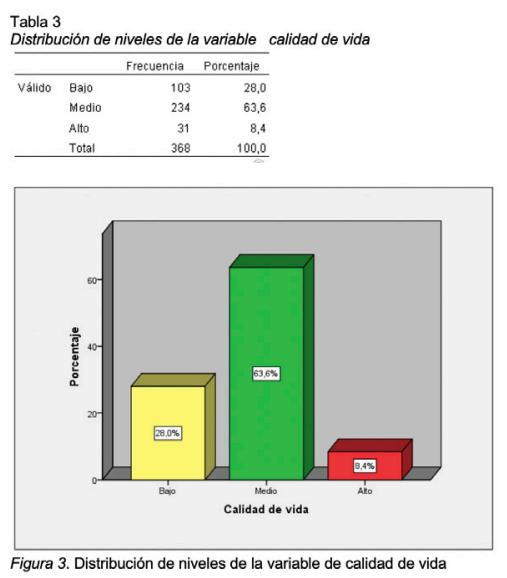

En la tabla 3 y figura 3 , se presentaron los niveles percibidos por los pobladores del distrito de Comas y con respecto a la variable calidad de vida, el $28 \%$ señaló como bajo, el $63,6 \%$ de medio y por último el $8,4 \%$ alto. Los habitantes de la zona 1 perciben a la calidad de vida en un nivel regular, por lo cual es necesario incidir, que esto se origina por el nivel regular tanto del soporte social brindado por los vecinos como por la construcción de las estructuras habitacionales de sus propio hogares.

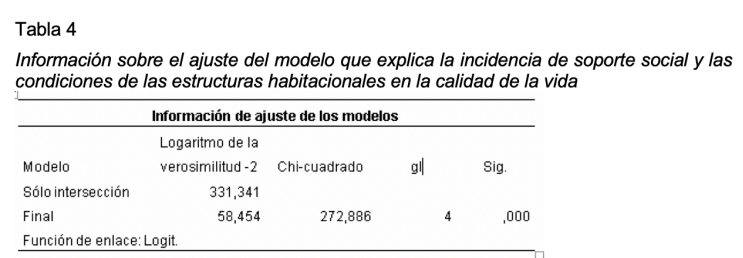

La prueba de contraste de la razón de verosimilitud señala que el modelo logístico es significativo $(x 2=272,886 ; p<0,05)$. Ello significa que el soporte social y las condiciones de las estructuras habitacionales inciden en la calidad de la vida de los pobladores

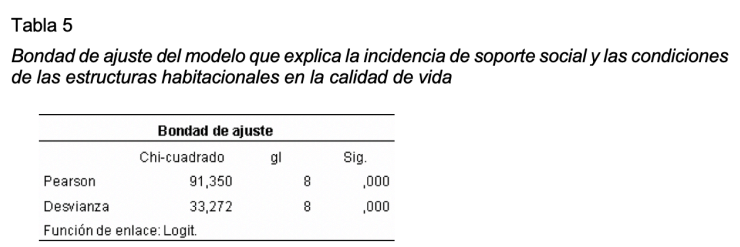

Una vez elaborado la muestra de regresión, es importante confirmar la calidad del ajuste de los valores predichos por el tipo a los valores observados. Se observa que la Desviación $(x 2=33,272)$ muestran un $p<0,05$; por tanto se puede señalar que el modelo de regresión donde se considera que el soporte social y las condiciones de las estructuras habitacionales son válidas y aceptables en la calidad del entorno de vida. 
Tabla 6

Psedo R - cuadrado del modelo que explica la incidencia de soporte social y las condiciones de las estructuras habitacionales en la calidad de la vida

\begin{tabular}{lc}
\hline \multicolumn{2}{c}{ Pseudo R cuadrado } \\
\hline Coxy Snell &, 524 \\
Nagelkerke &, 640 \\
McFadden| &, 435 \\
Función de enlace: Logit. \\
\hline
\end{tabular}

De acuerdo a la tabla 6, el Pseudo R cuadrado nos señala que el modelo está asociado con la variable dependiente, con una significatividad de $p=0,002$ y que la fuerza de las variables independientes (soporte social y las condiciones de las estructuras habitacionales) nos permite predecir la variable dependiente (calidad de la vida). Además, Cox y Snell indica el índice de 0,524, Nagelkerke indica el índice 0,640 y Mc Fadden 0,435. El R cuadrado de Nagelkerke establece la eficacia predictiva de la probabilidad de ocurrencia de las categorías de la variable dependiente en un $64 \%$, lo cual indica a su vez que el $36 \%$ restante viene explicado por las otras variables que no fueron incluidas en el modelo.

\section{Conclusiones}

\section{Primera}

A la luz de los resultados, se puede apreciar que existe incidencia de las condiciones del soporte social y las estructuras en la calidad de vida de las familias en la zona 1 Comas, 2019, ya que el modelo logístico es significativo $(p<0,05)$; se ajusta bien a los datos (Desviación con $p<0,05$ ); y explica el $64 \%$ de la variable dependiente la calidad de vida.

\section{Segunda}

A la luz de los resultados, se puede apreciar que existe incidencia del soporte social en el nivel regular y las condiciones de las estructuras habitacionales también en el nivel regular hacia la calidad del entorno de la vida de las familias en la zona 1 Comas, ya que el modelo logístico es significativo $(p<0,05)$; se ajusta bien a los datos (Desviación con $p<0,05$ ).

\section{Tercera}

A la luz de los resultados, se puede apreciar que existe la incidencia del soporte social y las condiciones de las estructuras habitacionales en la calidad de la acción de vida de las familias en la zona 1 Comas, precisando que la Municipalidad de Comas oriente a las familias sobre el mejoramiento de las futuras construcciones.

\section{Cuarta}

A la luz de los resultados, se puede apreciar que existe la incidencia del soporte social y las condiciones de las estructuras habitacionales en la calidad del resultado de vida de las familias en la zona 1 Comas, orientando a la población organizar soporte social, en la que se demuestre reciprocidad, disponibilidad y apoyo práctico; de manera que beneficie a la misma población, para elevar sus niveles de calidad de vida. 


\section{Referencias Bibliográficas}

Araujo, F. (2017). Diseño arquitectónico de viviendas progresivas de interés social para el barrio "menfis bajo", en la ciudad de Loja. Recuperado de: http:// repositorio.uide.edu.ec/bitstream/37000/2431/1/T-UIDE-0698.pdf

Haramoto, E. (2002). Un sistema de información en vivienda. Una proposición preliminar. Revista planes de desarrollo urbano. 16(44), 211-230.

Hernández, R., Fernández, R. y Baptista, P. (2014). Metodología de la investigación.

México: Mc Gram - Hill.

Janampa, R. (2018). Diseñoestructural de un edificio multifamiliarde 5 pisos yun semisótano,Ate - 2018. (Tesis de maestría). Universidad César Vallejo, Perú.Recuperadode:http://repositorio. ucv.edu.pe/bitstream/handle/UCV/35278/Janampa_CRM.pdf?seq uence=1\&isAllowed=y Jonkmann K., Thoemmes F., Lüdtke O. y Trautwein U. (2014). Personality traits and living arrangements in young adulthood: selection and socialization. Dev Psychol, 50(3):683-698. Recuperado de: DOI: 10.1037/a0034239.

Karstoft, K., Nielsen, T. y Nielsen, A. (2019). Measuring Social Support Among Soldiers With the Experienced Post-Deployment Social Support Scale (EPSSS): A Rasch-Based Construct Validity Study. Behavioral Medicine, 2(2). 122-134. Recuperado de: https://doi.org/10.1080/ 08964289.2019 .1676192

Landáruzi, M. y Mercado, J. (2004). Algunos factores físicos y psicológicos relacionados con la habitabilidad interna de la viviendall. Medio Ambiente y Comportamiento Humano. 5.(1,2). 231-249.Recuperadode: handle/11362/2376/S2003000_es.pdf.txt

Maquera, J. (2015). Conjunto residencial para reducir el déficit habitacional en el distrito y provincia de Tacna. (Tesis de maestría) Universidad privada de Tacna, Perú. Recuperado de: http://repositorio.upt.edu.pe/bitstream/UPT/85/1/maquera-turpo- jhony.pdf

OMS (1999). Vivienda saludable. Calidad de las condiciones de la vivienda y calidad de vida. Recuperado de: http://webcache.googleusercontent.com/ search?q=cache:5hXYJ2Tb5XAJ:www.bvs de.paho.org/bvsasv/e/iniciativa/posicion/siete. $\mathrm{pdf}+\& \mathrm{~cd}=1 \& \mathrm{hl}=\mathrm{es}-419 \& \mathrm{ct}=\mathrm{clnk} \& \mathrm{gl}=\mathrm{pe}$

Prieto, M. (2013). Condiciones habitacionales y calidad de vida urbana. El caso de la Ciudad de BahíaBlanca. Recuperado de: https://pdfs.semanticscholar.org/06a1/ c3cc2fe35e66601326d7622f5bb9c184cb1c.p df

Rashidah, N., Kaur, G., Ahmad, A. y Khalili, J. (2012). Housing Conditions and Quality of Life of the Urban Poor in Malaysia. Procedia-Social and Behavioral Sciences, 50(1); 827-833. Recuperado de: https://doi.org/10.1016/j.sbspro.2012.08.085

Rocha, P. (2008). Social Support and Social Network in Family Nursing: Reviewing Concepts. Rev Latinoam Enferm, 16(2). $32{ }^{2} 4$ - 337 . Recuperadode: http://www.scielo.br/scielo.php?script=sci_nlinks\&ref=000100\&pid=S01032100201200050002100001\&lng=en

Sánchez, H. y Reyes, C. (2015). Metodología y diseño de la investigación científica. Perú: 
San Marcos.

Stoppa, J. y Marchioro, N. (2012). Structure and Functionality of the Social Support Network for Adults with Cancer. Acta Paul. Enferm. 25(5). 231-245. Recuperado de: http://dx.doi. org/10.1590/S0103-21002012000500021

Wanzirah, H., Tusting, L., Arinatwe, E., Katureebe, A., Katureebe, A., Maxwell, J., Rek, J., Bottomley, S., Moses, K., Dorsey, G., y Lindsay, S. (2017). Mind the Gap: House Structure and the Risk of Malaria in Uganda. Plos One 10(1). 251-260. Recuperado de: https://doi. org/10.1371/journal.pone.0117396

Wu, H., Wu, S., Wu, H., Xia, Q. y Li, N. (2017). Living Arrangements and Health-Related Quality of Life in Chinese Adolescents Who Migrate from Rural to Urban Schools: Mediating Effect of Social Support. Int J Environ Res Public Healthv, 14(10). 371- 380. Recuperado de: https://dx.doi.org/10.3390\%2Fijerph 